\title{
ANÁLISE DE ÁCIDOS GRAXOS NÃO-ESTERIFICADOS DE PLASMA HUMANO POR CROMATOGRAFIA GASOSA CAPILAR COM INJEÇÃO SEM DIVISÃO DE FLUXO
}

\author{
Jacqueline G. Ney, Alexandre G. Torres e Nádia M. F. Trugo* \\ Departamento de Bioquímica, Instituto de Química, Universidade Federal do Rio de Janeiro, CT, Bloco A, Cidade Universitária, \\ 21949-900 Rio de Janeiro - RJ
}

Recebido em 3/6/03; aceito em 18/11/03; publicado na web em 27/05/04

\begin{abstract}
ANALYSIS OF NON-ESTERIFIED FATTY ACIDS IN HUMAN PLASMA BY CAPILLARY GAS-CHROMATOGRAPHY WITH SPLITLESS INJECTION. The aim of the present work was to test the combination of non-esterified fatty acid (NEFA) isolation using fumed silicon dioxide with capillary gas-chromatography (C-GC) with splitless injection for the analysis of NEFAs in human plasma. Injection volume, solvent re-condensation and split purge flow-rate were the parameters evaluated for the analysis of fatty acid methyl esters by C-GC. The use of a solvent re-condensation technique, associated with $1.0 \mu \mathrm{L}$ injection and a split purge flow rate of $80 \mathrm{~mL} / \mathrm{min}$ resulted in satisfactory analysis of NEFAs. Fourteen fatty acids were identified in plasma samples, ranging from 2.03 to $184.0 \mu \mathrm{mol} / \mathrm{L}$. The combination of both techniques proved useful for routine analyses of plasma NEFAs.
\end{abstract}

Keywords: non-esterified fatty acids; capillary gas-chromatography with splitless injection; fumed silicon dioxide.

\section{INTRODUÇÃO}

Os ácidos graxos desempenham importantes funções na fisiologia humana, tais como de substrato energético e na estrutura de membranas celulares ${ }^{1}$. Quando mobilizados do tecido adiposo, os ácidos graxos são transportados no plasma sanguíneo sob a forma não esterificada (ácidos graxos não-esterificados, AGNE), associados à albumina plasmática. Durante o jejum, o tecido adiposo sofre maior mobilização e os AGNE passam a ser o principal substrato energético para o organismo. Sua concentração plasmática total varia de 300 a $2000 \mu \mathrm{mol} / \mathrm{L}$, no período alimentado e no jejum, respectivamente ${ }^{1}$.

Para a determinação da composição dos AGNE é necessário primeiramente separá-los das demais classes de lipídios plasmáticos (triacilgliceróis, fosfolipídios, ésteres de colesterol, dentre outras). O método clássico usado para a separação das diferentes classes de lipídios é a cromatografia em camada delgada que, entretanto, apresenta algumas desvantagens, como elevado tempo de análise e potencial risco de oxidação dos ácidos graxos poli-insaturados ${ }^{2}$. Outros métodos de separação ou isolamento dos AGNE incluem a cromatografia em coluna ${ }^{3}$ e a separação em cartuchos de extração em fase sólida ${ }^{4}$. Entretanto, o preparo das colunas é trabalhoso e a reprodutibilidade dos resultados é dependente da habilidade no seu preparo. Já os cartuchos de extração em fase sólida disponíveis comercialmente apresentam custo relativamente elevado para análises de rotina. No método descrito por Polette et al..$^{5}$, os AGNE são separados das demais classes de lipídios plasmáticos pelo uso do dióxido de silício fundido ("fumed silicon dioxide", DSF) como agente delipidante. Após agitação do plasma com o DSF, forma-se um gel denso no qual as lipoproteínas são aprisionadas, enquanto os AGNE associados à albumina plasmática permanecem em solução na fase aquosa, que é facilmente separada por centrifugação. Este método, além de ser relativamente rápido e de baixo custo, melhora a recuperação dos analitos, quando comparado à cromatografia em camada delgada $^{5}$. A composição dos AGNE pode então ser determinada por cromatografia gasosa.

*e-mail: trugo@iq.ufrj.br
$\mathrm{Na}$ análise por cromatografia gasosa de alta resolução a etapa mais crítica, no que concerne à exatidão e precisão das análises, é a injeção ${ }^{6-8}$. Para a análise de compostos em quantidades traço, é necessário utilizar técnicas de injeção onde a maior parte dos analitos seja arrastada para a coluna pelo gás de arraste ${ }^{7,8}$. A injeção na coluna a frio, a injeção com vaporização por temperatura programável, a injeção direta e a injeção sem divisão de fluxo são as mais utilizadas ${ }^{7}$. Entretanto, os dois primeiros injetores não fazem parte da configuração usual dos aparelhos de cromatografia gasosa capilar (CG-C) que são, em geral, equipados com um injetor do tipo "split/splitless" (com/sem divisão de fluxo). Além disso, a injeção na coluna a frio é pouco utilizada para a análise de matrizes complexas, pois as substâncias de maior ponto de ebulição tendem a se acumular na coluna e podem gerar artefatos após algumas injeções, além de diminuir a vida útil da coluna ${ }^{7,9}$. Na análise de AGNE por CG-C, a injeção na coluna a frio apresenta resultados satisfatórios ${ }^{5}$, porém não é recomendada para análises sucessivas, em função da contaminação da porção inicial da coluna. Este problema pode ser resolvido com a injeção sem divisão de fluxo que, por sua vez, é suscetível a problemas como a perda de analitos, que pode ser ou não discriminatória, e o alargamento dos picos ${ }^{8}$.

Apesar dessas limitações, a injeção sem divisão de fluxo apresenta bom potencial para análises de rotina de ácidos graxos, uma vez estabelecidas as condições mais adequadas. Dentre os parâmetros mais $\operatorname{críticos}^{8}$ que podem afetar a análise utilizando a injeção sem divisão de fluxo estão o volume injetado, a temperatura inicial da coluna (recondensação do solvente), a vazão do gás de arraste, o volume (capacidade) do "liner" do injetor, o diâmetro interno da coluna, a temperatura do injetor, o tempo que o divisor de fluxo permanece fechado e a vazão de gás na saída do divisor de fluxo do injetor.

A injeção sem divisão de fluxo resulta em menor recuperação dos analitos quando comparada à injeção na coluna a frio, portanto a combinação do isolamento pelo DSF com a CG-C com injeção sem divisão de fluxo precisa ser testada. A combinação destas duas técnicas apresenta um bom potencial para análises de rotina de AGNE. No presente trabalho foram padronizadas as condições para análise de AGNE no plasma humano por CG-C utilizando injeção sem divisão de fluxo, combinada com o isolamento prévio dos AGNE dos demais 
lipídios plasmáticos pelo DSF. Os seguintes parâmetros da CG-C foram testados: volume total injetado, temperatura inicial da coluna (recondensação do solvente) e vazão no divisor de fluxo do injetor.

\section{MATERIAL E MÉTODOS}

Todo o material utilizado no procedimento foi apropriadamente lavado ${ }^{10}$. Tubos de vidro com tampa de rosca e septo de Teflon ${ }^{\circledR}$ foram utilizados para o isolamento dos AGNE e para a derivatização dos ácidos graxos.

\section{Amostras de plasma}

As amostras de sangue foram coletadas de quatro voluntários adultos, por punção venosa após jejum noturno (12 h) em tubos vacutainer $^{\circledR}$ (Becton \& Dickinson, Brasil) contendo Na-EDTA como anticoagulante. O plasma sanguíneo foi separado por centrifugação a $1500 \times g / 15 \mathrm{~min}$. Volumes iguais de cada amostra de plasma foram misturados e alíquotas da mistura foram armazenadas a $-20{ }^{\circ} \mathrm{C}$ até análise. Essas alíquotas foram utilizadas para avaliar a capacidade do DSF em isolar os AGNE e para determinar a precisão do método de análise de AGNE por CG-C, expressa como a variabilidade (coeficiente de variação, CV\%) intra- e inter-ensaio, utilizando 6 replicatas para cada análise.

\section{Isolamento dos AGNE plasmáticos pelo uso de DSF}

A separação dos AGNE plasmáticos das demais classes de lipídios foi realizada de acordo com o método descrito por Polette et al. ${ }^{5}$, empregando o DSF. A separação foi testada previamente, utilizando o dobro do volume de plasma (400 $\mu \mathrm{L})$ empregado no método original, com a finalidade de obter um extrato com maior concentração de AGNE. A capacidade do DSF de isolar os AGNE de um volume maior de plasma foi, então, avaliada por cromatografia em camada delgada.

Para o teste, $2,0 \mathrm{~mL}$ de uma suspensão aquosa $(4,1 \% ; \mathrm{m} / \mathrm{v}) \mathrm{de}$ DSF (Sigma, EUA, S5380) sob agitação magnética, foram adicionados a alíquotas de 200 e $400 \mu \mathrm{L}$ de plasma. Após agitação vigorosa em vórtex e centrifugação a $500 \times g / 13 \mathrm{~min}$, os lipídios do sobrenadante foram extraídos ${ }^{11}$. As mesmas quantidades de plasma foram também utilizadas para extração dos lipídios totais ${ }^{11}$, sem adição prévia de DSF. Os extratos foram concentrados com jato suave de $\mathrm{N}_{2}$ e aplicados em cromatoplaca de sílical gel G-60, com zona de concentração (Merck, Alemanha, ref. 11845), previamente ativada $\left(110^{\circ} \mathrm{C} / 1 \mathrm{~h}\right)$. Além dos extratos das amostras, foi aplicada na cromatoplaca uma mistura contendo os padrões comerciais de ácido palmítico (Sigma, EUA, ref. P-0500), tripalmitoilgicerol (Sigma, EUA, ref. P-5880), L- $\alpha$-dipalmitoil-fosfatidilcolina (Sigma, EUA, ref. P-6267) e palmitato de colesterila (Sigma, EUA, ref. C-6072). A cromatografia foi desenvolvida com a mistura de solventes benzina leve:éter etílico:ácido acético glacial (Merck, P.A., Alemanha), 80:20:1 (v/v/v). As bandas cromatográficas foram reveladas com solução de ácido sulfúrico (50\% v/v, Merck, P.A., Alemanha) e aquecimento em estufa $\left(120^{\circ} \mathrm{C}\right)$. Imediatamente, as bandas dos analitos dos extratos das amostras, tratadas ou não com DSF, foram identificadas por comparação com os Rf dos padrões comerciais. Esse teste foi repetido duas vezes.

\section{Análise de AGNE por cromatografia gasosa capilar (CG-C)}

Todas as análises foram realizadas em aparelho Shimadzu GC14B, equipado com injetor tipo "split/splitless", coluna Omegawax320 (polietileno-glicol, $30 \mathrm{~m}, 0,32 \mathrm{~mm}$ D.I., 0,25 $\mu \mathrm{m}$ de espessura de filme; Supelco, Co., EUA) e detector por ionização em chama. A temperatura do detector foi mantida em $280^{\circ} \mathrm{C}$. A pressão do gás de arraste (He) foi ajustada de modo a obter velocidade linear de $25,0 \mathrm{~cm} / \mathrm{s}(1,04 \mathrm{~mL} / \mathrm{min})$ que, de acordo com as curvas de van Deemter ${ }^{12}$, resulta em boa resolução dos picos cromatográficos. A vazão na purga do septo foi mantida em 1,0 $\mathrm{mL} / \mathrm{min}$ de modo a minimizar o risco de perda de analitos através da purga sem permitir, no entanto, que eventuais resíduos do septo permanecessem no injetor. A vazão de $0,5 \mathrm{~mL} / \mathrm{min}$ na purga do septo é suficiente para evitar o aparecimento de artefatos analíticos ${ }^{8}$. A temperatura do injetor (280 ${ }^{\circ} \mathrm{C}$ ) e a técnica de injeção com agulha quente ${ }^{8}$ foram previamente otimizadas na injeção com divisão de fluxo para análise sem discriminação dos analitos ${ }^{8,10,13}$. O "liner" utilizado (Supelco, Co., EUA, ref. 221-32544-00) tem volume interno de $600 \mu \mathrm{L}$. O divisor de fluxo (purga de "split") foi mantido fechado por 1min após a injeção, para assegurar a transferência dos analitos para a coluna.

\section{Estabelecimento das condições de análise por CG-C}

As condições cromatográficas foram estabelecidas utilizando uma mistura de padrões comerciais (Sigma, EUA), de proporção de massa conhecida, contendo os ésteres metílicos dos seguintes ácidos graxos: 8:0; 10:0; 12:0; 14:0; 16:0; 16:1(9); 18:2(9,12); 18:3(6,9,12); $18: 3(9,12,15) ; 20: 0 ; 20: 1(11) ; 20: 3(8,11,14) ; 20: 4(5,8,11,14) ; 22: 0$; $22: 1(13) ; 24: 0 ; 24: 1(15)$ e $22: 6(4,7,10,13,16,19)$. Os picos cromatográficos dos ésteres metílicos dos ácidos graxos do padrão foram utilizados como referência para avaliar a influência das condições testadas sobre a exatidão dos resultados das análises.

Os parâmetros testados na CG-C foram: (1) volume total injetado, $0,2 \mu \mathrm{L}$ de solução de padrões em $n$-hexano adicionados de 0,$2 ; 0,4$; 0,$6 ; 0,8$ e 1,0 $\mu \mathrm{L}$ de $n$-hexano (volumes totais de 0,$4 ; 0,6 ; 0,8 ; 1,0$ e $1,2 \mu \mathrm{L}$, com a mesma massa de padrões para cada injeção); (2) temperatura inicial da coluna, $180^{\circ} \mathrm{C}$ [Gradiente $1: 180^{\circ} \mathrm{C}\left(10^{\prime}\right)+2{ }^{\circ} \mathrm{C} / \mathrm{min}$ até $210^{\circ} \mathrm{C}\left(20^{\prime}\right)$ ] e $40^{\circ} \mathrm{C}$ [Gradiente 2: $40^{\circ} \mathrm{C}\left(2^{\prime}\right)+20^{\circ} \mathrm{C} / \mathrm{min}$ até $180^{\circ} \mathrm{C}\left(5^{\prime}\right)+2{ }^{\circ} \mathrm{C} /$ min até $210^{\circ} \mathrm{C}\left(20^{\prime}\right)$ ], sem e com re-condensação do solvente ( $n$-hexano), respectivamente e (3) vazão do gás de arraste na saída do divisor de fluxo do injetor: 40, 80 e $120 \mathrm{~mL} / \mathrm{min}$.

Todas as análises foram realizadas pelo menos em duplicata e os resultados apresentados são as médias aritméticas dos resultados experimentais.

\section{Análise de AGNE nas amostras de plasma}

Após confirmação do isolamento dos AGNE com DSF e estabelecimento das condições de análise por CG-C com os padrões comerciais dos ésteres metílicos de ácidos graxos, o procedimento de determinação dos AGNE foi avaliado empregando as amostras de plasma. Seis alíquotas de $400 \mu \mathrm{L}$ das amostras foram transferidas para tubos de vidro de fundo cônico, às quais foram adicionados $25 \mu \mathrm{L}$ de uma solução de ácido heptadecanóico (padrão interno; Sigma, EUA, ref. H-3500) 0,2 mg/mL de etanol absoluto. Em seguida procedeu-se ao isolamento dos AGNE das demais classes de lipídios plasmáticos com o DSF, conforme descrito no item Isolamento dos AGNE plasmáticos pelo uso de DSF, exceto que o sobrenadante contendo os AGNE foi liofilizado. O resíduo seco contendo os AGNE foi derivatizado ${ }^{14}$. Resumidamente, o resíduo foi ressuspenso em 2,0 mL de metanol (para cromatografia, Tedia, EUA) e transferido para tubos de hidrólise com rosca esmerilhada (Schott, Alemanha). Após adição de $500 \mu \mathrm{L}$ de $n$-hexano (para cromatografia, Fluka, EUA), adicionaram-se lentamente $100 \mu \mathrm{L}$ de cloreto de acetila (Merck, Alemanha, ref. 100031) sob agitação suave. Os ácidos graxos foram metilados em banho maria a $80^{\circ} \mathrm{C} / 30 \mathrm{~min}$. Após neutralização da solução com 1,5 mL de $\mathrm{NaHCO}_{3}$ (P.A., Merck) $10 \%$ (m/v), os ésteres metílicos resultantes foram extraídos com $500 \mu \mathrm{L}$ de $n$-hexano, que foi evaporado com jato suave de $\mathrm{N}_{2}$. Os analitos foram ressuspen- 
sos em $25 \mu \mathrm{L}$ de $n$-hexano e armazenados em atmosfera de $\mathrm{N}_{2}$ a $-20{ }^{\circ} \mathrm{C}$ por até uma semana.

As amostras foram, então, analisadas por CG-C nas condições anteriormente estabelecidas com os padrões. Os ésteres metílicos dos ácidos graxos das amostras foram identificados por comparação de seus tempos de retenção relativos $\left(r_{18: 0}\right)^{15}$, com os tempos de retenção relativos de padrões comerciais: mistura descrita acima e mistura comercial ("Menhaden oil", Supleco, EUA, ref. 47085-U). As áreas dos picos dos ácidos graxos identificados foram corrigidas pelos fatores de correção teóricos (Ft') de Ackman e Sipos ${ }^{16,17}$. As áreas assim corrigidas foram utilizadas para os cálculos das concentrações relativas $(\mathrm{g} \%, \mathrm{~m} / \mathrm{m}$; normalização interna) e absolutas ( $\mu \mathrm{mol} / \mathrm{L}$; padronização interna) dos ácidos graxos nas amostras de plasma.

\section{RESULTADOS E DISCUSSÃO}

\section{Isolamento dos AGNE plasmáticos pelo uso de dióxido de silício fundido (DSF)}

O método de isolamento dos AGNE utilizando o DSF não requer o uso de solventes orgânicos, é simples, relativamente rápido e de menor custo do que os demais métodos para separação dos AGNE. A técnica clássica de separação por cromatografia em camada delgada requer tempo relativamente elevado para concluir todas as etapas da análise (ativação das cromatoplacas, aplicação das amostras, corrida cromatográfica, revelação e recuperação das bandas cromatográficas) e os analitos permanecem parte deste tempo em contato com o ar atmosférico, o que pode expor os ácidos graxos poliinsaturados a um maior risco de oxidação ${ }^{2}$.

A quantidade de DSF (82 mg) utilizada no método descrito por Polette et al. ${ }^{5}$ para delipidar $200 \mu \mathrm{L}$ de plasma foi igualmente eficiente para $400 \mu \mathrm{L}$ de plasma, pois os extratos tratados com DSF obtidos a partir de ambos os volumes de plasma apresentaram na cromatografia em camada delgada apenas a banda correspondente aos ácidos graxos não esterificados, com $\operatorname{Rf}$ de 0,24, igual ao do ácido palmítico, utilizado como padrão. Os Rfs obtidos para os demais padrões utilizados, tripalmitoilgicerol, palmitato de colesterila e L- $\alpha$-dipalmitoilfosfatidilcolina, foram $0,68,1,04 \mathrm{e} 0$, respectivamente. A vantagem de não aumentar o volume da suspensão de DSF proporcionalmente ao volume de plasma é evitar o aumento do tempo de liofilização dos extratos. Portanto, o procedimento utilizando $82 \mathrm{mg}$ de DSF e o volume de $400 \mu \mathrm{L}$ de plasma foi adotado para proceder ao isolamento dos AGNE das amostras, para posterior análise em CG-C com injeção sem divisão de fluxo. A possibilidade de analisar maior volume de plasma permite a identificação de um maior número de analitos.

\section{Estabelecimento das condições de análise de ácidos graxos por CG-C}

As áreas dos picos de todos os ésteres metílicos da mistura de padrões aumentaram em função do aumento do volume injetado, atingindo valor máximo para o volume de 1,0 $\mu \mathrm{L}$ (Figura 1). Esta relação entre volume injetado e área dos picos foi estudada, para cada um dos analitos em separado, por ánalises de regressão nãolinear (programa GraphPad Prism v 2.0, GraphPad Software Inc., EUA) e pode ser explicada pela seguinte equação matemática:

Área $=$ Área $_{\min } \times e^{\alpha \cdot v o l}$

onde Área ${ }_{\min }$ é o menor valor de área para determinado padrão; $\alpha$ é uma constante; vol é o volume injetado. As regressões apresentaram coeficiente de determinação $\left(r^{2}\right)$ médio de $0,97( \pm 0,07)$. É provável que outros modelos matemáticos exponenciais explicassem melhor a relação entre volume injetado e área dos picos, entretanto isto não pode ser determinado devido ao pequeno número de pontos (quatro) na região entre 0,4 e 1,0 $\mu \mathrm{L}$, o que reduz os graus de liberdade das análises de regressão. Quando o volume de 1,2 $\mu \mathrm{L}$ foi injetado, a área dos picos foi menor do que para a injeção de $1,0 \mu \mathrm{L}$, para todos os analitos da mistura de padrões.

Esses resultados sugerem que a quantidade de vapor do solvente formado no injetor aquecido com a injeção de $1,2 \mu \mathrm{L}$ pode ter superado a capacidade volumétrica do "liner" do injetor, ocasionando perda dos analitos. Esta perda ocorreu provavelmente pela purga do septo do injetor, já que a massa de cada um dos EMAG foi a mesma para as injeções dos cinco volumes testados. A relação entre volume injetado e área dos picos pode ser causada pela função entre o volume injetado e a eficiência da transferência dos analitos para a coluna, descrita para análises com injeção com divisão de fluxo ${ }^{8}$. Quanto maior o volume injetado, menos o gás de arraste se mistura com o vapor do solvente e, por conseguinte, maior é a eficiência do efeito de re-condensação ${ }^{8}$, favorecendo a transferência dos analitos para a coluna. Desta forma, para a injeção sem divisão de fluxo, a eficiência de transferência dos analitos parece aumentar exponencialmente com o aumento do volume injetado até certo limite, quando a perda de analitos por ultrapassar a capacidade volumétrica do "liner" prevalece.
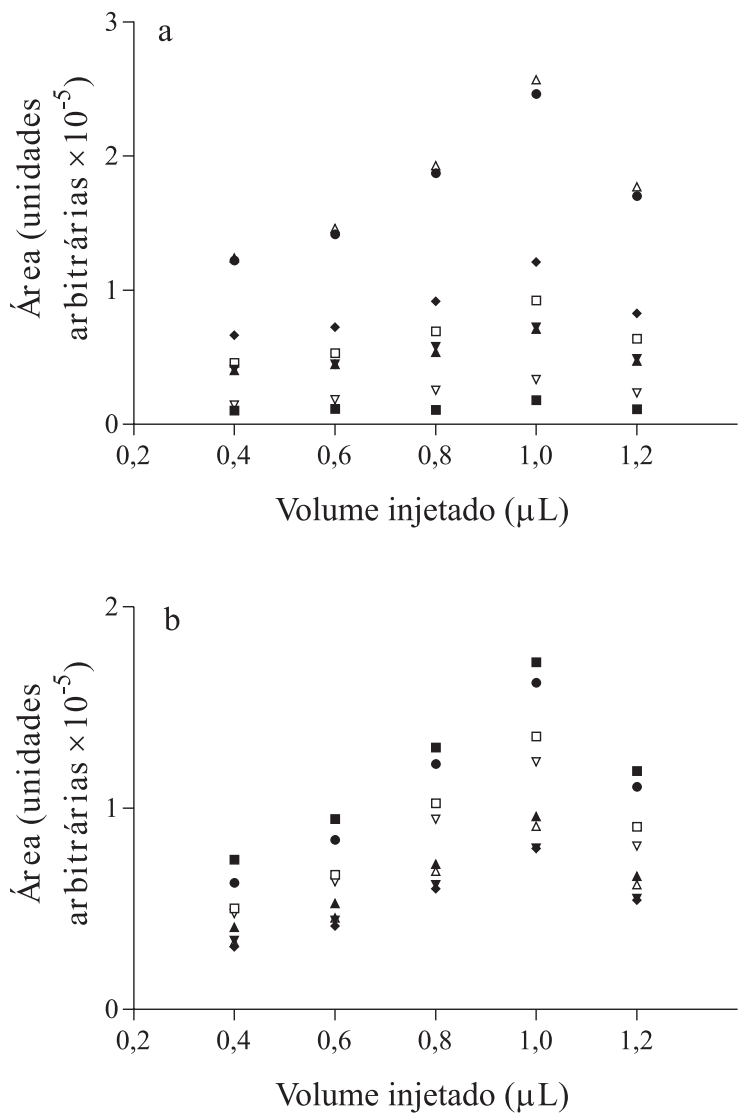

Figura 1. Efeito do volume de injeção sobre as áreas dos picos dos padrões de ésteres metílicos dos ácidos graxos (mistura de padrões): a- ésteres metílicos: $\mathbf{\square}$ - 12:0; $\boldsymbol{\Delta}$ - 14:0; $\boldsymbol{\nabla}$ - 16:0; - 16:1(9); ○- 18:2(9,12); $\square-18: 3(6,9,12) ; \triangle-18: 3(9,12,15) ; \nabla-20: 0 ; \boldsymbol{b}$ - ésteres metílicos: - 20:1(11); $\boldsymbol{\Delta}$ - 20:3(8,11,14); $\boldsymbol{\nabla}-20: 4(5,8,11,14) ;-22: 0 ; \bullet-22: 1(13)$; - 24:0; $\triangle$ - 24:1(15) e $\nabla$ - 22:6(4,7,10,13,16,19). Condições cromatográficas: vazão da purga do septo- $1,0 \mathrm{~mL} / \mathrm{min}$; vazão na purga do divisor de fluxo do injetor- $120 \mathrm{~mL} / \mathrm{min}$; temperatura do injetor e do detector (DIC)- $280{ }^{\circ} \mathrm{C}$; gradiente de temperatura da coluna- $180{ }^{\circ} \mathrm{C}\left(10^{\prime}\right)+2{ }^{\circ} \mathrm{C} /$ min até $210^{\circ} \mathrm{C}\left(20^{\prime}\right)$ 
A reprodutibilidade do volume injetado é um fator crítico para a identificação dos analitos de interesse, uma vez que a variação no volume injetado pode afetar negativamente a precisão do tempo de retenção ${ }^{9}$. Desta maneira, deve-se evitar injetar volumes muito reduzidos. Para a análise de amostras diluídas deve-se injetar o maior volume possível. Portanto, adotou-se o volume de $1,0 \mu \mathrm{L}$, por ser o maior volume entre os testados que não causou perda dos analitos vaporizados no injetor. A partir de então, as demais injeções foram realizadas com $0,8 \mu \mathrm{L}$ de amostra ou padrão $+0,2 \mu \mathrm{L}$ de $n$-hexano.

Segundo $\mathrm{Grob}^{8}$, quando o injetor é operado sem divisão de fluxo, tende a ocorrer espalhamento das bandas cromatográficas, ocasionando picos menores e com a base mais larga, prejudicando sua resolução como no cromatograma da Figura 2a. Este problema é causado pelo elevado tempo de transferência dos analitos para a coluna. Este intervalo de tempo pode causar e/ou agravar problemas associados à injeção sem divisão de fluxo, como o alargamento das bandas cromatográficas e a discriminação no injetor ${ }^{8}$. A técnica de re-condensação do solvente, quando a temperatura da coluna no início da corrida é mantida em $20-30{ }^{\circ} \mathrm{C}$ inferior ao ponto de ebulição do solvente (P.E. $n$-hexano $=69^{\circ} \mathrm{C}$ ), apesar de gerar uma corrida mais longa, pode evitar esses problemas pois acelera a transferência dos analitos para a coluna ${ }^{8}$. Portanto, testaram-se os efeitos da temperatura inicial da coluna sobre alguns parâmetros qualitativos e quantitativos da análise. Na Figura 2 são apresentados cromatogramas típicos obtidos após análise de mistura de padrões sem (Gradiente 1, Figura 2a) e com (Gradiente 2, Figura 2b) re-condensação do solvente. No cromatograma da Figura $2 b$, os picos apresentam resolução visivelmente superior ao cromatograma da Figura 2a.

Para uma avaliação mais objetiva do efeito da re-condensação do solvente sobre os resultados, os seguintes parâmetros foram determinados: o somatório das áreas dos picos (unidades arbitrárias), a largura da frente do solvente (s) e o fator de separação $(\alpha)^{18}$ entre os dois primeiros picos do cromatograma (Tabela 1). De acordo com o parâmetro quantitativo (somatório das áreas dos picos) e com os parâmetros qualitativos (largura da frente de solvente e $\alpha$ ) analisados, a técnica de re-condensação do solvente mostrou-se mais apropriada para a análise dos ésteres metílicos de ácidos graxos com injeção sem divisão de fluxo. A melhor recuperação dos analitos observada subjetivamente nos cromatogramas (Figura 2, a e b) é confirmada pelo somatório das áreas dos picos, que é duas vezes maior com o uso da técnica de re-condensação do solvente (Gradiente 2, Tabela 1). A melhor separação proporcionada pelo uso da técnica de re-condensação do solvente possibilita análises mais exatas, por minimizar o risco de co-eluição dos analitos e por tornar a integração das áreas dos picos mais exata, além de possibilitar a identificação de maior número de ácidos graxos, potencialmente presentes em amostras biológicas.

A vazão de gás na saída do divisor de fluxo do injetor foi também avaliada. Na análise sem divisão de fluxo do injetor, o divisor de fluxo permanece fechado por um período de tempo suficiente para garantir a transferência dos analitos para a coluna, após o qual o divisor de fluxo é aberto para eliminar o vapor de solvente que permanece no injetor. A vazão do gás deve ser suficiente para purgar rapidamente o solvente vaporizado que ainda permanece no compartimento do injetor e deve ser avaliada quanto ao efeito potencial na discriminação dos analitos ${ }^{8}$, a qual afeta a quantificação dos EMAG. Portanto, foi testada a influência da vazão do gás na purga do divisor de fluxo do injetor sobre a análise quantitativa dos EMAG.

Para a comparação entre as três vazões testadas, as concentrações relativas dos EMAG, após correção pelos $\mathrm{Ft}^{17}$, foram comparadas com sua proporção real de massa na solução de padrões (Figura 3). As diferenças relativas (resíduos relativos, \%) entre a concentração de cada EMAG determinada experimentalmente $(\mathrm{g} \%, \mathrm{~m} / \mathrm{m})$ e
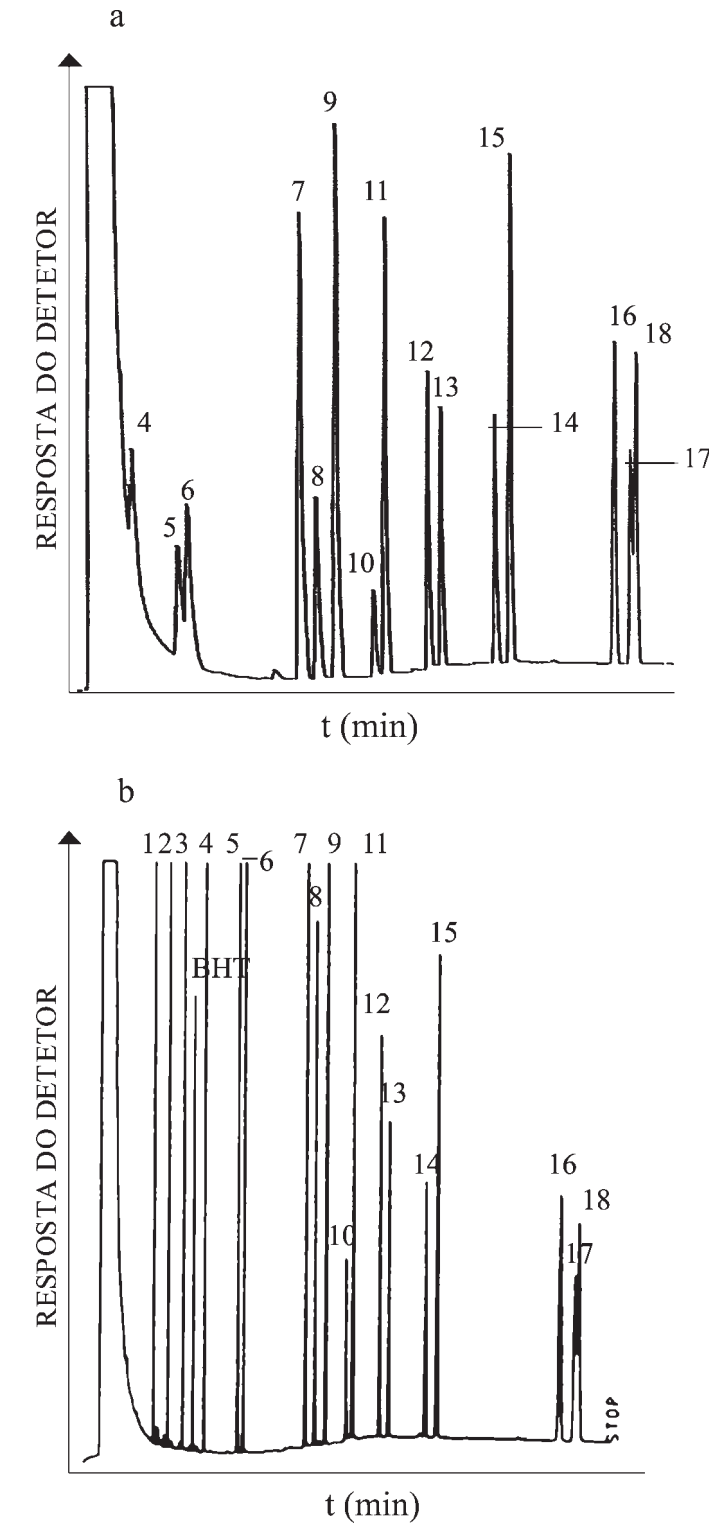

Figura 2. Cromatogramas típicos das análises de ésteres metílicos de ácidos graxos (mistura de padrões) por cromatografia gasosa capilar com injeção sem divisão de fluxo, sem (a) e com (b) o uso da técnica de re-condensação do solvente: $\boldsymbol{a}$ - Gradiente $1: 180{ }^{\circ} \mathrm{C}\left(10^{\prime}\right)+2{ }^{\circ} \mathrm{C} / \mathrm{min}$ até $210{ }^{\circ} \mathrm{C}\left(20^{\prime}\right)$. $\boldsymbol{b}$ Gradiente 2: $40{ }^{\circ} \mathrm{C}\left(2^{\prime}\right)+20^{\circ} \mathrm{C} / \min$ até $180^{\circ} \mathrm{C}\left(5^{\prime}\right)+2{ }^{\circ} \mathrm{C} / \mathrm{min}$ até $210^{\circ} \mathrm{C}$ (20’). Identificação dos picos dos ésteres metílicos: 1- 8:0; 2- 10:0; 3- 12:0; 4- 14:0; 5- 16:0; 6- 16:1(9); 7- 18:2(9,12); 8-18:3(6,9,12); 9- 18:3(9,12,15); 10- 20:0; 11 - 20:1(11); 12- 20:3(8,11,14); 13-20:4(5,8,11,14); 14- 22:0; 15- 22:1(13); 16- 24:0; 17-24:1(15) e 18-22:6(4,7,10,13,16,19). Condições cromatográficas: vazão da purga do septo- $1,0 \mathrm{~mL} / \mathrm{min}$; vazão na purga do divisor de fluxo do injetor- $120 \mathrm{~mL} / \mathrm{min}$; temperatura do injetor e do detector (DIC)- $280{ }^{\circ} \mathrm{C}$; volume injetado- $1,0 \mu \mathrm{L}$

sua proporção real de massa foram calculadas de acordo com a equação:

Resíduo relativo $(\%)=\left(\mathrm{C}_{\mathrm{t}}-\mathrm{C}_{\mathrm{p}}\right) \times 100 / \mathrm{C}_{\mathrm{p}}$

onde $\mathrm{C}_{\mathrm{t}}$ é a concentração $(\mathrm{g} \%, \mathrm{~m} / \mathrm{m})$ do analito na vazão testada e $\mathrm{C}_{\mathrm{p}}$ é a proporção real de massa do analito na solução de padrões.

Dentre as vazões testadas (60, 80 e $120 \mathrm{~mL} / \mathrm{min})$, a vazão de $80 \mathrm{~mL} /$ min no divisor de fluxo do injetor resultou em concentrações relativas 
Tabela 1. Influência da re-condensação do solvente da amostra sobre parâmetros qualitativos e quantitativos da análise de ésteres metílicos de ácidos graxos por cromatografia gasosa capilar com injeção sem divisão de fluxo

\begin{tabular}{lcc}
\hline Parâmetro & $\begin{array}{c}\text { Sem re-condensação } \\
\text { do solvente } \\
\text { na coluna } \\
\text { Gradiente 1 }\end{array}$ & $\begin{array}{c}\text { Com re-condensação } \\
\text { do solvente } \\
\text { na coluna } \\
\text { Gradiente } 2\end{array}$ \\
\hline $\begin{array}{l}\text { Somatório das áreas } \\
\text { dos picos cromatográficos a } \\
\text { (unidades arbitrárias) }\end{array}$ & 1.467 .967 & 2.802 .846 \\
$\begin{array}{l}\text { Largura da frente de } \\
\text { solvente (s) }\end{array}$ & 32,7 & 24,6 \\
Fator de separação $(\alpha)^{\mathrm{b}}$ & 1,05 & 1,16 \\
\hline
\end{tabular}

${ }^{\mathrm{a}}$ Inclui todos os picos com exceção da frente do solvente; ${ }^{\mathrm{b}} \alpha=\left[\left(\mathrm{t}_{\mathrm{r} 2}-\right.\right.$ $\left.\left.\mathrm{t}_{\mathrm{M}}\right) / \mathrm{t}_{\mathrm{M}}\right] /\left[\left(\mathrm{t}_{\mathrm{r} 1}-\mathrm{t}_{\mathrm{M}}\right) / \mathrm{t}_{\mathrm{M}}\right]$. Gradiente $1: 180^{\circ} \mathrm{C}\left(10^{\prime}\right)+2{ }^{\circ} \mathrm{C} / \mathrm{min}$ até $210^{\circ} \mathrm{C}$ (20'); Gradiente 2: $40^{\circ} \mathrm{C}\left(2^{\prime}\right)+20^{\circ} \mathrm{C} / \mathrm{min}$ até $180{ }^{\circ} \mathrm{C}\left(5^{\prime}\right)+2{ }^{\circ} \mathrm{C} /$ min até $210^{\circ} \mathrm{C}\left(20^{\prime}\right)$. Condições cromatográficas: vazão na purga do septo- $1,0 \mathrm{~mL} / \mathrm{min}$; vazão na purga do divisor de fluxo do injetor$120 \mathrm{~mL} / \mathrm{min}$; temperatura do injetor e do detector (DIC)- $280{ }^{\circ} \mathrm{C}$; volume injetado- $1,0 \mu \mathrm{L}$.

dos padrões mais similares à proporção real de massas para todos os EMAG, como apresentado na Figura 3. As vazões de 60 e $120 \mathrm{~mL} /$ min resultaram em discriminação dos EMAG de mais baixa $(8: 0 ; 10: 0$; $12: 0 ; 14: 0)$ e mais elevada $[24: 0 ; 24: 1(15)$ e $22: 6(4,7,10,13,16,19)]$ massa molar. Os resíduos relativos para a vazão de $80 \mathrm{~mL} / \mathrm{min}$ variaram até $\pm 10 \%$ da referência para todos os EMAG, resultando em uma análise quantitativa satisfatória. A média dos módulos dos resíduos relativos foi de 10,5; 3,3 e 7,1 para as vazões de 60, 80 e $120 \mathrm{~mL} / \mathrm{min}$, respectivamente. Portanto, a vazão do gás na saída do divisor de fluxo do injetor foi ajustada em $80 \mathrm{~mL} / \mathrm{min}$ e confirmada diariamente, a fim de assegurar a regularidade da vazão durante as análises das amostras e garantir-lhes precisão e exatidão.

Dentre as condições cromatográficas que conhecidamente ${ }^{8}$ podem afetar as análises por CG-C com injeção sem divisão de fluxo, a

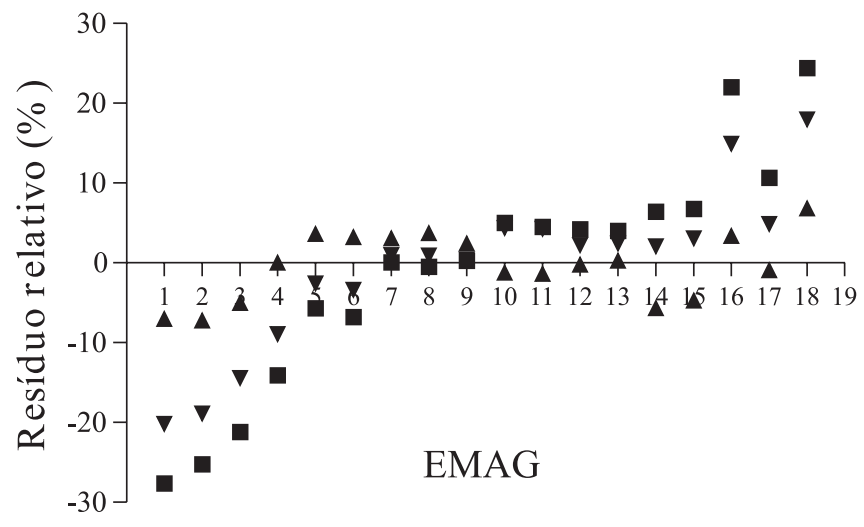

Figura 3. Influência da vazão de gás na purga do divisor de fluxo do injetor sobre a análise quantitativa dos ésteres metílicos de ácidos graxos (EMAG; mistura de padrões). Vazão do gás ajustada a: $\mathbf{\square}$ 6; $\boldsymbol{\Delta} 80$ e $\boldsymbol{\nabla} 120 \mathrm{~mL} / \mathrm{min}$. Numeração dos EMAG segue padrão da Figura 2. Resíduos relativos (\%) calculados de acordo com a Equação (2). Condições cromatográficas: vazão da purga do septo- 1,0 mL/min; temperatura do injetor e do detector (DIC)$280{ }^{\circ} \mathrm{C}$; volume injetado $-1,0 \mu \mathrm{L}$; gradiente de temperatura da coluna$40{ }^{\circ} \mathrm{C}\left(2^{\prime}\right)+20^{\circ} \mathrm{C} / \mathrm{min}$ até $180^{\circ} \mathrm{C}\left(5^{\prime}\right)+2{ }^{\circ} \mathrm{C} / \mathrm{min}$ até $210^{\circ} \mathrm{C}\left(20^{\prime}\right)$

otimização do volume de injeção, da temperatura inicial da coluna (re-condensação do solvente) e da vazão do gás na saída do divisor de fluxo do injetor mostraram-se suficientes para a obtenção de resultados satisfatórios na análise de AGNE.

\section{Análise de AGNE no plasma}

Foram identificados 14 ácidos graxos, cujas concentrações individuais (Tabela 2) e total $(719 \pm 66,7 \mu \mathrm{mol} / \mathrm{L})$ estão dentro da faixa esperada para plasma humano ${ }^{2,5,19}$. Os coeficientes de variação (CV\%) do método de análise dos AGNE em plasma por CG-C utilizando injetor sem divisão de fluxo foram calculados com base nas concentrações relativas $(\mathrm{g} \%, \mathrm{~m} / \mathrm{m})$ dos ácidos graxos identificados nas replicatas das amostras de plasma. A variabilidade na quantificação

Tabela 2. Concentrações absolutas $(\mu \mathrm{mol} / \mathrm{L})$ e relativas $(\mathrm{g} \%, \mathrm{~m} / \mathrm{m})$ dos ácidos graxos não-esterificados do plasma sanguíneo e variabilidade (CV\%) do método de análise dos AGNE

\begin{tabular}{|c|c|c|c|c|}
\hline AGNE & $\begin{array}{c}\text { Concentração Absoluta } \\
n=11^{\mathrm{a}} \\
(\mu \mathrm{mol} / \mathrm{L}, \text { média } \pm \mathrm{s})\end{array}$ & $\begin{array}{c}\text { Concentração Relativa } \\
\mathrm{n}=11^{\mathrm{a}} \\
(\mathrm{g} \%, \text { média } \pm \mathrm{s})\end{array}$ & $\begin{array}{l}\text { Variabilidade } \\
\text { Intra-ensaio } \\
\mathrm{n}=6^{\mathrm{b}}(\mathrm{CV} \%)^{\mathrm{c}}\end{array}$ & $\begin{array}{l}\text { Variabilidade } \\
\text { Inter-ensaio } \\
\mathrm{n}=6^{\mathrm{b}}(\mathrm{CV} \%)^{\mathrm{c}}\end{array}$ \\
\hline $15: 0$ & $3,16 \pm 0,23$ & $0,39 \pm 0,02$ & 5,9 & 4,2 \\
\hline $16: 0$ & $221 \pm 19,6$ & $28,7 \pm 0,97$ & 3,4 & 2,9 \\
\hline 16:1(9) & $22,7 \pm 2,60$ & $2,94 \pm 0,30$ & 11 & 8,2 \\
\hline $18: 2(9,12)$ & $157 \pm 22,5$ & $22,2 \pm 1,55$ & 5,3 & 4,8 \\
\hline $18: 3(9,12,15)$ & $5,73 \pm 0,56$ & $0,81 \pm 0,05$ & 6,6 & 4,7 \\
\hline $20: 1(11)$ & $3,44 \pm 0,54$ & $0,53 \pm 0,09$ & 20 & 12 \\
\hline $20: 3(8,11,14)$ & $4,38 \pm 0,73$ & $0,68 \pm 0,07$ & 7,4 & 8,0 \\
\hline $20: 4(5,8,11,14)$ & $15,9 \pm 2,87$ & $2,43 \pm 0,28$ & 9,7 & 7,4 \\
\hline $20: 5(5,8,11,14,17)$ & $2,35 \pm 0,43$ & $0,36 \pm 0,05$ & 7,4 & 16 \\
\hline
\end{tabular}

${ }^{a}$ Inclui os resultados das análises das replicatas das amostras de plasma utilizadas para os cálculos da variabilidade intra- e inter-ensaio; ${ }^{\mathrm{b}}$ replicatas das amostras de plasma utilizadas para os cálculos de variabilidade; ${ }^{\mathrm{c}}$ calculado a partir da média e estimativa do desvio padrão (s) da concentração relativa $(\mathrm{g} \%, \mathrm{~m} / \mathrm{m})$. 
dos ácidos graxos que pode ser atribuída ao método foi inversamente proporcional à concentração dos ácidos graxos na amostra, como era esperado. O coeficiente de variação inter-ensaio dos ácidos graxos saturados variou entre 2,9 e 4,2\%, dos mono-insaturados entre 1,6 e $12 \%$ e dos poli-insaturados entre 4,7 e $16 \%$, enquanto que o coeficiente de variação intra-ensaio dos ácidos graxos saturados variou de 3,4 a $12 \%$, e dos mono-insaturados e poli-insaturados variou de 5,3 a $20 \%$ e de 5,3 a $9,7 \%$, respectivamente.

As médias ponderadas dos coeficientes de variação intra- e interensaio foram 5,4 e 3,5\%, respectivamente. Esta variabilidade média total pode ser considerada satisfatória uma vez que se refere a todo o método de análise, ou seja, inclui o isolamento dos AGNE pelo uso do DSF, a derivatização dos ácidos graxos e a análise por CG-C propriamente dita. O limite de quantificação do método foi cerca de $2 \mu \mathrm{mol} / \mathrm{L}$, uma vez que com esta concentração a reprodutibilidade foi $\leq 15 \%^{20}$ (Tabela 2 ).

\section{CONCLUSÃO}

A eficiência da etapa de isolamento dos analitos é fundamental para minimizar a presença de interferentes na análise cromatográfica de matrizes complexas, como o plasma sanguíneo, sendo especialmente importante para a análise de pequenas massas como os AGNE. O DSF mostrou-se adequado como agente delipidante do plasma no isolamento dos AGNE, na proporção de $82 \mathrm{mg}$ para $400 \mu \mathrm{L}$ de plasma. Este método é adequado para análises de rotina, em função do baixo custo do sorvente, do menor tempo dedicado ao isolamento dos AGNE e, especialmente, em função do uso da injeção sem divisão de fluxo, que evita o acúmulo rápido de compostos de elevado ponto de ebulição na coluna.

A padronização das condições cromatográficas, consideradas limitantes no uso do injetor sem divisão de fluxo, resultou na separação e recuperação satisfatória dos analitos de interesse. Aliado a isso, o menor tempo de permanência dos analitos no injetor, atribuído à técnica de re-condensação, minimiza a discriminação entre os AGNE. Os parâmetros cromatográficos testados (volume injetado, recondensação do solvente e vazão de gás na saída do divisor de fluxo do injetor) foram suficientes para minimizar ou eliminar os problemas associados à análise por cromatografia gasosa capilar sem divisão de fluxo do injetor e obter resultados satisfatórios.

Os resultados obtidos no presente trabalho mostram que o método proposto, utilizando pela primeira vez a combinação do isola- mento dos AGNE com DSF e a análise por CG-C com injeção sem divisão de fluxo, é apropriado para análises de rotina de ácidos graxos não-esterificados de plasma sanguíneo.

\section{AGRADECIMENTOS}

Os autores agradecem o auxílio financeiro do CNPq, CAPES, FAPERJ e as bolsas concedidas pelo CNPq (IC: J. G. Ney; Doutorado: A. G. Torres e Produtividade: N. M. F. Trugo).

\section{REFERÊNCIAS}

1. Newsholme, E. A.; Leech, A. R.; Biochemistry for the Medical Sciences, John Wiley \& Sons Ltd.: Chichester: UK, 1983.

2. Püttman, M.; Krug, H.; von Achsenstein, E.; Kattermann, R.; Clin. Chem. 1993, 39, 825

3. Ingalls, S.T.; Kriaris, M.S.; Xu, Y.; DeWulf, D.W.; Tserng, K.Y.; Hoppel, C.L.; J. Chromatogr. 1993, 619, 9

4. Burdge, G.C.; Wright, P.; Jones, A.E.; Wootton, S.A.; Br. J. Nutr. 2000 , 84, 781 .

5. Polette, A.; Durand, P.; Floccard, B.; Blache, D.; Anal. Biochem. 1992, 206, 241.

6. Eder, K.; J. Chromatogr., B: Biomed. Appl. 1995, 671, 113.

7. Klee, M.S. In: Modern Practice of Gas Chromatography, $3^{\text {rd }}$ ed.; Grob R. L., Ed.; John Wiley \& Sons: New York, 1995, cap. 8, p. 469-502.

8. Grob, K.; Split and Splitless Injection in Capillary Gas Chromatography, $3^{\text {rd }}$ ed.; Hüthig Buch Verlag GmbH: Heidelberg, Germany, 1993.

9. Rood, D.; A Practical Guide to the Care, Maintenance and Troubleshooting of Capillary Gas Chromatographic Systems; Hüthig Buch Verlag GmbH: Heidelberg, Germany, 1991.

10. Torres, A. G.; Trugo, N. M. F. ; Trugo, L. C.; J. Agric. Food Chem. 2002, $50,4156$.

11. Bligh, E.G.; Dyer, W.J.; Can. J. Biochem. Physiol. 1959, 37, 911.

12. Klee, M. S. In: Modern Practice of Gas Chromatography, $3^{\text {rd }}$ ed.; Grob R.L., Ed.; John Wiley \& Sons: New York, 1995, cap.4, p. 255-264.

13. Bannon, C.D.; Craske, J.D.; Felder, D.L.; Garland, I.J.; Norman, L.M.; J. Chromatogr. 1987, 407, 231.

14. Lepage, G.; Roy C.C.; J. Lipid Res. 1986, 27, 114

15. Ackman, R. G.; Methods Enzymol. 1969, 14, 329.

16. Ackman, R. G.; Sipos, J. C.; J. Am. Oil Chem. Soc. 1964, 41, 377.

17. Wolff, R. L.; Bayard, C. C.; Fabien, R. J.; J. Am. Oil Chem. Soc. 1995, 72,1471

18. Snyder L. R.; Kirkland J. J.; Introduction to Modern Liquid Chromatography, $2^{\text {nd }}$ ed.; John Wiley \& Sons: New York, 1979.

19. Fielding, B.A.; Reid, G.; Grady, M.; Humphreys, S.M.; Evans, K.; Frayn, K.N.; Br. J. Nutr. 2000, 83, 597.

20. Causon, R.; J. Chromatogr., B: Biomed. Appl. 1997, 689, 175. 